\title{
Identification of proteins in the aqueous humor associated with cataract development using iTRAQ methodology
}

\author{
MINHONG XIANG $^{1}$, XINGRU ZHANG $^{1,2}$, QINGSONG LI $^{1}$, HANMIN WANG $^{1}$, \\ ZHENYONG ZHANG $^{1}$, ZHUMEI HAN ${ }^{1}$, MEIQING KE ${ }^{1}$ and XINGXING CHEN ${ }^{1}$ \\ ${ }^{1}$ Department of Ophthalmology, Putuo Hospital, Shanghai University of Traditional Chinese Medicine, \\ Shanghai 200062; ${ }^{2}$ Department of Ophthalmology, Liqun Hospital, Shanghai 200333, P.R. China
}

Received February 4, 2016; Accepted January 26, 2017

DOI: $10.3892 / \mathrm{mmr} .2017 .6345$

\begin{abstract}
Proteins in the aqueous humor (AH) are important in the induction of cataract development. The identification of cataract-associated proteins assists in identifying patients and predisposed to the condition and improve treatment efficacy. Proteomics analysis has previously been used for identifying protein markers associated with eye diseases; however, few studies have examined the proteomic alterations in cataract development due to high myopia, glaucoma and diabetes. The present study, using the isobaric tagging for relative and absolute protein quantification methodology, aimed to examine cataract-associated proteins in the $\mathrm{AH}$ from patients with high myopia, glaucoma or diabetes, and controls. The results revealed that 445 proteins were identified in the $\mathrm{AH}$ groups, compared with the control groups, and 146, 264 and 130 proteins were differentially expressed in the three groups of patients, respectively. In addition, 44 of these proteins were determined to be cataract-associated, and the alterations of five randomly selected proteins were confirmed using enzyme-linked immunosorbent assays. The biological functions of these 44 cataract-associated proteins were analyzed using Gen Ontology/pathways annotation, in addition to protein-protein interaction network analysis. The results aimed to expand current knowledge of the pathophysiologic characteristics of cataract development and provided a panel of candidates for biomarkers of the disease, which may assist in further diagnosis and the monitoring of cataract development.
\end{abstract}

\section{Introduction}

Cataracts account for the most common cause of blindness worldwide, affecting over half $(51.8 \%)$ of all individuals with

Correspondence to: Dr Xingru Zhang, Department of Ophthalmology, Putuo Hospital, Shanghai University of Traditional Chinese Medicine, 164 Lanxi Road, Putuo, Shanghai 200062, P.R. China E-mail: zhangxingru928@hotmail.com

Key words: aqueous humor, cataract, diabetes, glaucoma, high myopia, proteome blindness, which renders it one of the most prevalent eye diseases (1). It has been accepted that age is the most important risk factor for cataracts, followed by environmental and genetics factors. Currently, there are three primary types of age-related cataracts, which differ in optical age, size, density, shape, color and location (2). The cataracts most associated with age are cortical cataracts, in which the initial opacity is confined to the outer lens shells. Cortical cataracts are more common in individuals aged $>65$ years (3). In nuclear cataracts, the opacity first appears in the center of the lens, which is the second most prevalent group. The age at which nuclear cataracts are most common is also $>65$ years. The third types of cataracts are posterior subcapsular cataracts (PSC), a less common group, which lie in the outermost layers of the cortex at the posterior pole.

In the last few decades, an increasing number of studies have been performed to investigate the developmental mechanism of cataracts to identify potential prevention and treatment strategies (4-6). Sufficient evidence has demonstrated that, in addition to aging, genetics and environmental factors, including UV exposure and X-ray irradiation, are involved in cataract development. For example, Ji et al (2015) found that prolonged UV exposure can increase cataract risk (7). Other environmental factors, including diabetes, glaucoma and myopia, are also important in cataract development (8). For example, diabetes is reported to be associated with age-related PSCs and cortical cataracts, but not nuclear cataracts (9). However, cigarette smoking has consistently been confirmed as a risk factor for nuclear cataracts, and possibly PSCs, but not cortical cataracts. In another previous report, diabetes was identified to be associated with the development of PSCs, and cigarette smoking may increase the risk of the formation of nuclear cataracts (10). The additional effects of these factors in cataract development have also been investigated. For example, in a report by Pan et al (2013), myopia, particularly high myopia, was determined to predispose to PSCs (11).

To date, proteome analyses have been used in various ocular diseases, including diabetic retinopathy, glaucoma and cataracts. It allows the simultaneous identification of a large number of proteins at a given time in specific cells, tissues or body fluids, which aims to identify proteomic peptide biomarkers of disease for the diagnosis of eye diseases, and elucidating the disease mechanism for providing novel 
therapies (12-16). For example, Izzotti et al (6) investigated the association between aqueous humor $(\mathrm{AH})$ protein content and the pathogenesis of open-angle glaucoma (POAG). It was determined that certain proteins underwent marked variation, which was associated with pathogenetic events characterizing POAG, including oxidative damage, mitochondrial damage, neural degeneration and apoptosis. These results not only indicated that proteomic analysis of the $\mathrm{AH}$ is a novel tool for POAG diagnosis, but provided an improved understanding of the mechanisms involved in the pathogenesis of POAG.

There have been several proteomic investigations of ocular fluids, including $\mathrm{AH}$, using two-dimensional gel electrophoresis (2-DE). However, this traditional technology is known to be poor for analyzing low quantities of protein (17). However, one type of high throughput technology, termed isobaric tagging for relative and absolute protein quantification (iTRAQ) labeling combined with mass spectrometry (MS) has been development for protein identification and relative quantification (18). Therefore, the present study aimed to take advantage of this technology to perform proteomic profiling of $\mathrm{AH}$ from three types of patient and their controls using iTRAQ combined with reversed-phase liquid chromatography (RPLC)/RPLC-MS. The results revealed marked proteomic differences between the controls and patients with high myopia, glaucoma or diabetes. Of note, 44 cataract-associated proteins were determined, five of which were randomly selected and identified using enzyme-linked immunosorbent assays (ELISA). The biological functions of these 44 cataract-associated proteins were analyzed using Gene Ontology (GO)/pathway annotation. Protein-protein interaction network analysis was also performed to incorporate known evidence of physical/functional interactions. The results aimed to provide potential $\mathrm{AH}$ biomarkers and offer insights into the mechanisms underlying cataract development associated with myopia, glaucoma and diabetes.

\section{Materials and methods}

Subjects. A total of 24 subjects in four groups were recruited for the present study, including six patients with high myopia, six patients with glaucoma, six patients with diabetes and six control patients (Table I). All patients were of Asian ethnicity and all patients with glaucoma were determined to have POAG, whose intraocular pressure was controlled between 10 and $21 \mathrm{~mm} \mathrm{Hg}$ by topical intraocular pressure-lowering medications. The cup-to-disc ratios of these patients were significantly increased, and they had early and middle visual field defects. The patients with diabetes were treated via oral administration of drugs to control blood sugar levels $(<6.1 \mathrm{mM} / \mathrm{l})$, with no microangiopathy and no insulin therapy. The control groups were age-related patients with cataracts but without glaucoma, high myopia or diabetes.

None of the subjects had a history or slit-lamp evidence of ocular trauma, nor use of systemic anti-metabolites, immunosuppressants or corticosteroids. No ocular diseases, other than cataracts, were present to meet inclusion criteria. The average age of these patients was $\sim 72$ years (range 59-88 years) and that of the control subjects was 71 years (range 60-81 years). Other characters of these subjects, including axial length and clinical application of the lens opacities classification system III (19), are demonstrated in Table I. The study protocol was reviewed and approved by the Ethics Committee of Putuo Hospital, Shanghai University of Traditional Chinese Medicine (Shanghai, China) and informed written consent was obtained from the patients.

Surgical sample preparation and $i T R A Q$ labeling. The collection and preparation of $\mathrm{AH}$ was performed by the same operator in a manner similar to that described in a previous report (10). All samples collected were digested with trypsin and labeled using iTRAQ according to the manufacturer's protocol (Applied Biosystems; Thermo Fisher Scientific, Inc., Waltham, MA, USA). The labeled digests were injected onto a 2.1x150 mm XBridge BEH 300 column (Waters Corporation, Milford, MA, USA) on a Shimadzu LC-20AD (Shimadzu Corporation, Kyoto, Japan) with UV detection at 214/280 nm. High-performance liquid chromatography solvent A consisted of $20 \mathrm{mM}$ ammonium formate in water (pH 10) and solvent $\mathrm{B}$ consisted of $20 \mathrm{mM}$ ammonium formate in water $(\mathrm{pH} \mathrm{10)}$ with $100 \%$ ACN. Peptides were separated at a flow rate of $200 \mu 1 / \mathrm{min}$ and eluted from the column with a 5 min gradient from 0 to $5 \%$ solvent B, followed by a 35 min gradient from 5 to $35 \%$ solvent B, a 5 min gradient from 35 and $80 \%$ solvent B, a 5 min gradient of $80 \%$ solvent $\mathrm{B}$, and a final $1 \mathrm{~min}$ gradient of from 80 to $0 \%$ solvent $\mathrm{B}$, followed by termination for $9 \mathrm{~min}$. A total of 20 gradient-based fractions were collected and were reduced using rotation vacuum concentrators (Christ RVC 2-25; Martin Christ Gefriertrocknungsanlagen $\mathrm{GmbH}$, Osterode am Harz, Germany), following which they were reconstituted to $50 \mu \mathrm{l}$ mixtures containing $5 \% \mathrm{ACN}$ and $0.1 \%$ ammonium formate.

Protein identification and quantification. MS was performed on a Triple TOF 5600 (Applied Biosystems; Thermo Fisher Scientific, Inc.) coupled with an Eksigent 1D plus with a ZORBAX 300SB-C18 column $(5 \mu \mathrm{m} ; 300 \AA$; 0.1x150 mm; Agilent Technologies, Inc., Santa Clara, CA, USA). Solvents for LC-MS separation of mixed peptides were as follows: Solvent A consisted of 5\% ACN and $0.1 \%$ ammonium formate; solvent B consisted of $95 \% \mathrm{ACN}$ and $0.1 \%$ ammonium formate. The peptides were eluted from the analytical column with a 65 min gradient ranging from 5 to $45 \%$ solvent B, followed by a 2 min gradient from 45 to $80 \%$ solvent $\mathrm{B}$, a $5 \mathrm{~min}$ gradient for $80 \%$ solvent $\mathrm{B}$, a 3 min gradient from 80 to $5 \%$ solvent $\mathrm{B}$, and a final $10 \mathrm{~min}$ gradient from 5 to $0 \%$ solvent B. Triple TOF 5600 MS was performed in data-dependent mode to automatically switch between MS and MS/MS acquisition. The MS spectra were obtained across the mass range of $350-1,250 \mathrm{~m} / \mathrm{z}$ in high resolution mode using a $250 \mathrm{msec}$ accumulation time per spectrum. Tandem MS were scanned from 100 to $1,250 \mathrm{~m} / \mathrm{z}$ in high sensitivity mode with rolling collision energy. The 20 most intense precursors were selected for fragmentation per cycle with a dynamic exclusion time of 9 sec. Protein identification was performed according to the following parameters: Cysteine alkylation, iodoacetic acid; ID focus, 
Table I. Summary of human aqueous humor samples.

\begin{tabular}{|c|c|c|c|c|c|}
\hline Group (Labeled ID) & Patient ID & Gender & Age (Years old) & Axial length & LOCSIII \\
\hline \multirow{6}{*}{ Controls (117) } & 1 & Male & 73 & 25.29 & 3 \\
\hline & 2 & Female & 78 & 24.78 & 4 \\
\hline & 3 & Female & 81 & 22.64 & 4 \\
\hline & 4 & Female & 63 & 23.88 & 3 \\
\hline & 5 & Male & 60 & 23.73 & 3 \\
\hline & 6 & Male & 72 & 23.56 & 3 \\
\hline \multirow[t]{6}{*}{ Patients with high myopia (119) } & 1 & Male & 68 & 26.22 & 3 \\
\hline & 2 & Male & 70 & 27.3 & 3 \\
\hline & 3 & Female & 65 & 29.87 & 3 \\
\hline & 4 & Male & 85 & 25.87 & 4 \\
\hline & 5 & Female & 59 & 26.34 & 3 \\
\hline & 6 & Female & 63 & 27.65 & 4 \\
\hline \multirow[t]{6}{*}{ Patients with glaucoma (118) } & 1 & Female & 75 & 22.61 & 3 \\
\hline & 2 & Female & 59 & 22.84 & 3 \\
\hline & 3 & Female & 81 & 23.81 & 4 \\
\hline & 4 & Male & 79 & 22.78 & 3 \\
\hline & 5 & Female & 76 & 23.21 & 3 \\
\hline & 6 & Male & 83 & 23.52 & 4 \\
\hline \multirow[t]{6}{*}{ Patients with diabetes (121) } & 1 & Female & 70 & 23.68 & 3 \\
\hline & 2 & Female & 78 & 24.35 & 4 \\
\hline & 3 & Male & 76 & 23.75 & 3 \\
\hline & 4 & Female & 62 & 24.65 & 3 \\
\hline & 5 & Female & 68 & 22.78 & 3 \\
\hline & 6 & Male & 88 & 23.65 & 4 \\
\hline
\end{tabular}

LOCSIII, lens opacities classification system III.

biological modification; digestion, trypsin; species: Homo; database: Swissprot human (177,396 entries; http://www. expasy.ch/sprot/); search effort, thorough ID. Protein Pilot software version 4.5 (ABSciex, Foster City, CA, USA) was used for relative quantification of proteins.

ELISA. The alterations in the expression levels of five randomly selected proteins were confirmed using ELISA (JRDun Biotech, Shanghai, China). These five proteins were isoform 5 of chordin-like protein 1 (CRDL1), amyloid-like protein 2 (APLP2), dickkopf-related protein 3 (F6SYF8), cytochrome P450 2S1 (CP2S1) and fibulin-1 (F8W7M9). The ELISA was performed according to the manufacturer's protocol of the kits (cat. nos. DRE13425, DRE10132, DRE12755, DRE12757 and DRE12481). A total of three repeat determinations were performed for all samples.

Bioinformatics analyses. The 44 differently expressed proteins were annotated according to the GO database (http://www.geneontology.org/). The pathway annotations of proteins were established by searching against the Kyoto Encyclopedia of Genes and Genomes database (http://www. genome.jp/kegg/pathway.html). In addition, the functional regulatory networks of the proteomics-identified proteins were analyzed using the Search Tool for the Retrieval of Interacting Genes/Proteins (STRING; version 9.0; http://string.embl.de/) database. Statistical analyses, including independent t-tests were performed using SPSS 17.0 software (SPSS, Inc., Chicago, IL, USA. P $\leq 0.05$ was considered to indicate a statistically significant difference.

\section{Results}

Proteome analysis of human AHs. To perform comprehensive proteomic profiling of human $\mathrm{AH}$ in the present study, $\mathrm{AH}$ samples were collected from patients with high myopia, glaucoma, diabetes and controls. Using an iTRAQ methodology, a total of 445 proteins were identified in these patients, which revealed the broadest human $\mathrm{AH}$ proteome, compared with those in previous studies $(20,21)$. Among these proteins, 334 proteins have previously been reported in eyes in the $\mathrm{AH}$, cornea, vitreous humor, tears, choroid and retina, whereas the remaining 111 proteins were novel, according to the human eye proteome project (22). Of note, for 224 proteins, it was the first time they have been detected in $\mathrm{AH}$, showing the value of this proteomic profiling technology in examining human AHs. Further functional classification revealed that the 445 proteins identified reflected the most common and abundant proteins in human AHs, the functions of which included complement, 


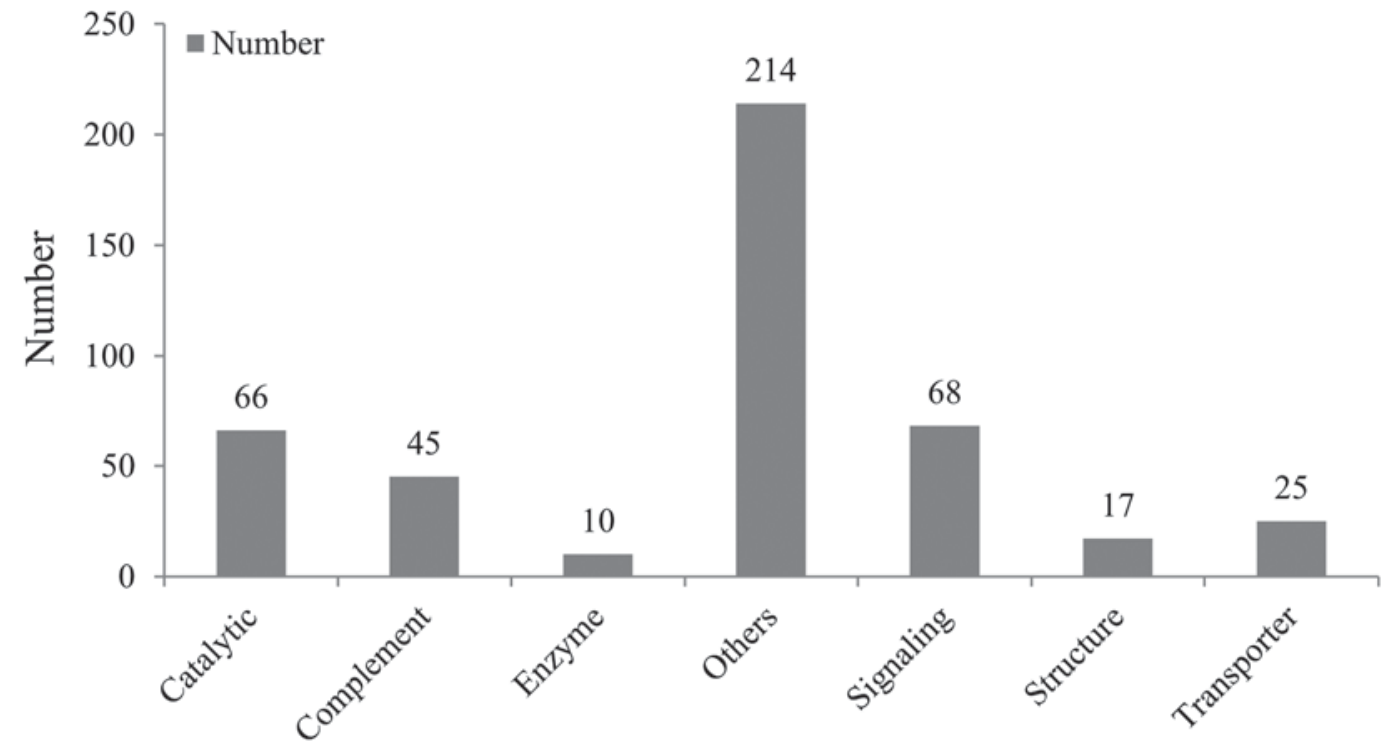

Figure 1. Functional distribution of the 445 proteins identified in aqueous humor samples.
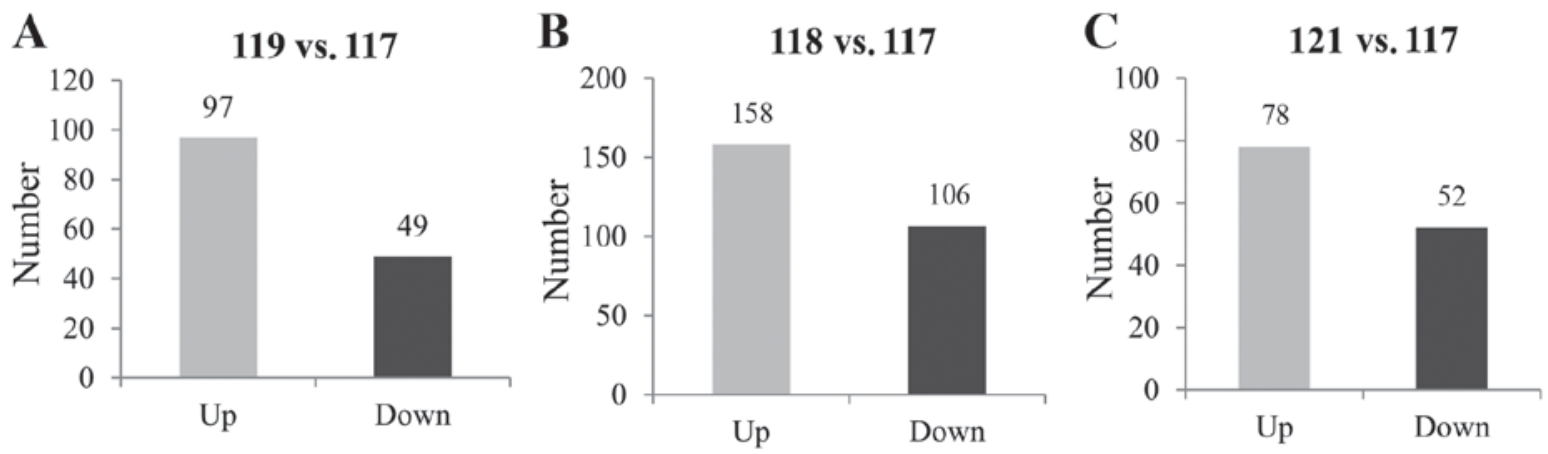

Figure 2. Differences between patients and controls. 117, controls; (A) 119 patients with high myopia; (B) 118 patients with glaucoma; (C) 121 patients with diabetes. Up, upregulated; Down, downregulated.

signaling, catalytic, enzyme, structural and transporter functions (Fig. 1).

Proteomic alterations in the AH from patients with high myopia. Previous reports have demonstrated that myopia is closely associated with cataracts. Accordingly, the present study performed a proteomic analysis of the AH composition between patients with high myopic eyes and controls with non-myopic cataracts to identify the possible mechanism underlying cataract development. The result revealed that there were 146 differently expressed proteins (Fig. 2A). Among the proteins, 49 proteins were downregulated, including the probable phospholipid-transporting ATPase IK, which was the most markedly altered, and was involved in acrosome reactions and binding of spermatozoa to zonapellucida. Two proteins belonging to the keratin family, keratin, type II cytoskeletal 2 (K22E) and keratin, type I cytoskeletal 10 (K1C10) were also included, which are markers for various pathways of keratinocyte differentiation (23). By contrast, 97 proteins were upregulated in patients with high myopia. Notably, six crystalline proteins, including $\alpha$-crystallin B chain, $\beta$-crystallin A4, $\alpha$-crystallin A chain, $\beta$-crystallin B1, $\beta$-crystallin B2 and $\beta$-crystallin A3, were upregulated, and these are known to be critical for lens clarity and refraction, and involved in cataract formation $(24,25)$. The levels of four keratins, keratin, type II cytoskeletal 6B (K2C6B), keratin, type II cytoskeletal 6A (K2C6A), keratin, type I cytoskeletal 14 (K1C14) and keratin, type I cytoskeletal 16 (K1C16) were also increased.

Proteomic alterations of AH from patients with glaucoma. Previous studies have also revealed that cataract progression frequently follows glaucoma (26). To determine the possible mechanism underlying cataract formation due to glaucoma, the presentstudy performed a comparative proteomic analysis of $\mathrm{AH}$ between patients with glaucoma and controls. As demonstrated in Fig. 2B, a total of 264 proteins were differently expressed, including 158 upregulated proteins and 106 downregulated proteins. Similarly, six keratin proteins (type I and II) were found in patients with glaucoma, whereas four keratins [K1C10, keratin, type I cytoskeletal 9 (K1C9), keratin, type II cytoskeletal 1 (K2C1) and $\mathrm{K} 22 \mathrm{E}$, were downregulated, and keratin, type II cytoskeletal 5 (K2C5) and K2C6A] were upregulated $>2.14$-fold. In addition, all the crystalline proteins were upregulated, with the exception of $\beta$-crystallin A3. Of note, two lysozyme $\mathrm{C}$ proteins were upregulated the most, with fold-changes $>195$, and these had a bacteriolytic function. 
Table II. Proteomic changes of aqueous humor related to cataract development.

\begin{tabular}{|c|c|c|c|}
\hline Accession no. & Average (119/117) & Average (118/117) & Average $(121 / 117)$ \\
\hline splP01871|IGHM_HUMAN & 0.10 & 0.42 & 0.22 \\
\hline splP35908IK22E_HUMAN & 0.20 & 0.15 & 0.58 \\
\hline splP13645|K1C10_HUMAN & 0.23 & 0.05 & 0.27 \\
\hline trlF6SYF8IF6SYF8_HUMAN & 0.40 & 0.28 & 0.43 \\
\hline splQ08629|TICN1_HUMAN & 0.44 & 0.25 & 0.56 \\
\hline splP01833|PIGR_HUMAN & 0.46 & 64.78 & 3.63 \\
\hline splP26447IS10A4_HUMAN & 0.53 & 0.52 & 0.29 \\
\hline splQ9NQ79-3ICRAC1_HUMAN & 0.54 & 0.21 & 0.57 \\
\hline splQ9HCB6ISPON1_HUMAN & 0.57 & 0.37 & 0.30 \\
\hline splP81605IDCD_HUMAN & 1.53 & 7.48 & 2.90 \\
\hline splP07451ICAH3_HUMAN & 1.63 & 6.17 & 3.19 \\
\hline splQ16378IPROL4_HUMAN & 1.76 & 14.52 & 1.97 \\
\hline splP05543ITHBG_HUMAN & 1.81 & 1.67 & 1.51 \\
\hline splP08185|CBG_HUMAN & 1.90 & 1.51 & 1.86 \\
\hline splP25311|ZA2G_HUMAN & 1.90 & 8.09 & 1.98 \\
\hline splP02760|AMBP_HUMAN & 1.96 & 44.48 & 1.91 \\
\hline splP02774-3IVTDB_HUMAN & 2.02 & 1.56 & 2.20 \\
\hline splP07357|CO8A_HUMAN & 2.08 & 1.78 & 1.96 \\
\hline trlB0YIW2IB0YIW2_HUMAN & 2.27 & 8.87 & 1.71 \\
\hline splP01024|CO3_HUMAN & 2.46 & 2.18 & 2.36 \\
\hline tr|C9JEU5IC9JEU5_HUMAN & 2.46 & 15.86 & 4.08 \\
\hline splP01610|KV118_HUMAN & 2.49 & 4.92 & 2.62 \\
\hline splP02675|FIBB_HUMAN & 2.67 & 20.09 & 2.36 \\
\hline splP01877|IGHA2_HUMAN & 2.70 & 2.22 & 3.64 \\
\hline splP01861IIGHG4_HUMAN & 2.71 & 0.40 & 2.94 \\
\hline splP04217|A1BG_HUMAN & 2.77 & 2.62 & 2.05 \\
\hline trlC9JV77IC9JV77_HUMAN & 2.92 & 5.30 & 1.72 \\
\hline splP02748|CO9_HUMAN & 3.15 & 4.70 & 1.77 \\
\hline splP19652|A1AG2_HUMAN & 3.24 & 3.29 & 2.73 \\
\hline splP01031|CO5_HUMAN & 3.79 & 7.59 & 3.35 \\
\hline splP02763|A1AG1_HUMAN & 4.00 & 8.90 & 5.61 \\
\hline splP01042-2|KNG1_HUMAN & 4.00 & 8.01 & 1.86 \\
\hline splP02649|APOE_HUMAN & 4.01 & 11.09 & 2.27 \\
\hline splP43652IAFAM_HUMAN & 4.01 & 2.74 & 1.62 \\
\hline splP01011IAACT_HUMAN & 4.33 & 6.95 & 1.51 \\
\hline trlE7EMM4|E7EMM4_HUMAN & 5.02 & 8.10 & 3.80 \\
\hline splP02743ISAMP_HUMAN & 5.07 & 8.65 & 2.01 \\
\hline trlB7ZKJ8IB7ZKJ8_HUMAN & 5.34 & 6.97 & 1.66 \\
\hline trlV9GYM3IV9GYM3_HUMAN & 7.21 & 3.73 & 1.84 \\
\hline splP01009|A1AT_HUMAN & 7.47 & 13.49 & 2.21 \\
\hline splP01008IANT3_HUMAN & 10.80 & 21.10 & 4.46 \\
\hline splP02489|CRYAA_HUMAN & 18.01 & 2.56 & 1.75 \\
\hline splP08779|K1C16_HUMAN & 20.77 & 2.56 & 0.59 \\
\hline splP53673|CRBA4_HUMAN & 21.37 & 3.46 & 2.11 \\
\hline
\end{tabular}

117, controls; 119, patients with high myopia; 118, patients with glaucoma; 121, patients with diabetes.

Proteomic alterations of AH from patients with diabetes. There is considerable evidence that diabetes is associated with increased oxidative stress, which may be prone to cataract formation (27). Therefore, the present study determined the differently expressed proteins between patients with diabetes and controls to identify the proteomic alterations. As demonstrated in Fig. 2C, 130 proteins were differently expressed. Among these proteins, the levels of 78 proteins 
A

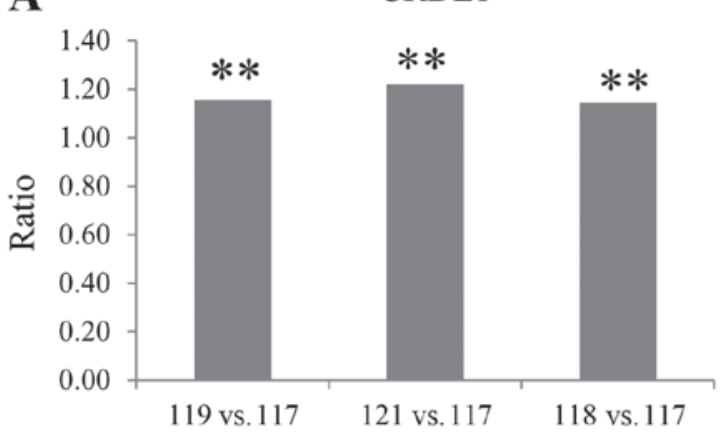

C

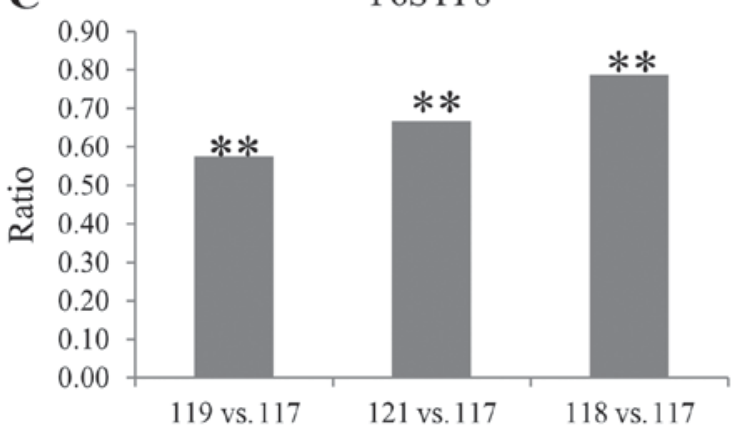

B

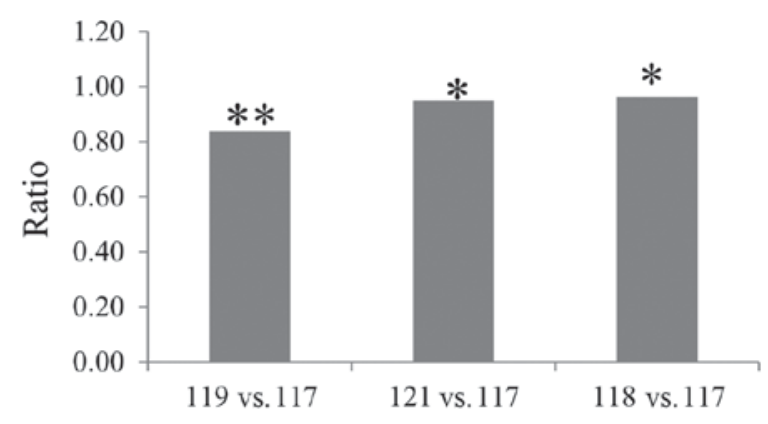

D

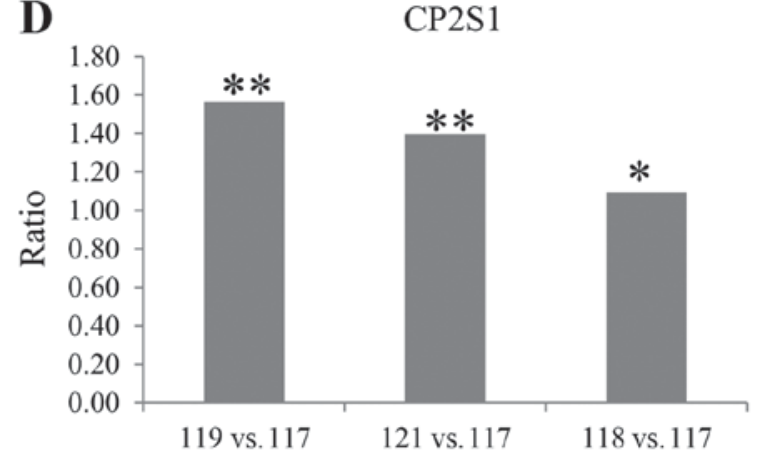

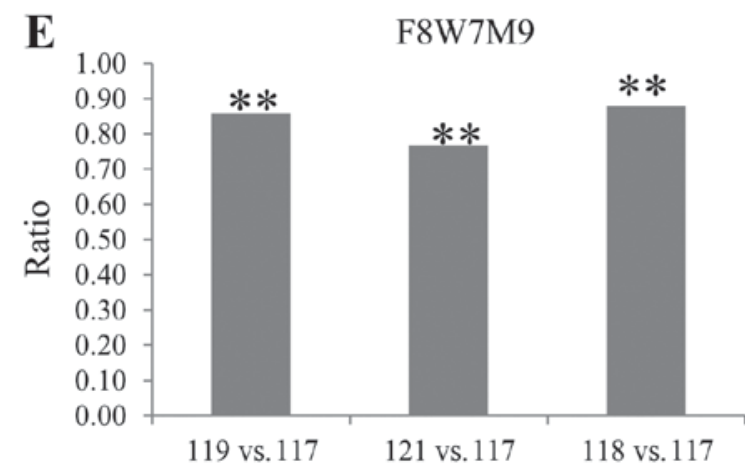

Fold change

Figure 3. ELISA assay of (A-E) five proteins showing altered expression. ${ }^{* *} \mathrm{P}<0.01 ;{ }^{*} \mathrm{P}<0.05 .117$, controls; 119 patients with high myopia; 118 patients with glaucoma; 121 patients with diabetes. CRDL1, chordin-like protein 1; APLP2, amyloid-like protein 2; F6SYF8, dickkopf-related protein 3; CP2S1, cytochrome P450 2S1; F8W7M9, fibulin-1.

were upregulated, although only one keratin protein (K2C6B) was upregulated. Similarly, the expression levels of all three crystallin proteins, including $\beta$-crystallin B1 (CRBB1), $\alpha$-crystallin A chain (CRYAA) and $\beta$-crystallin A4 (CRBA4) were increased. By contrast, 52 proteins were downregulated in the patients with diabetes, including the five keratins, $\mathrm{K} 1 \mathrm{C} 10, \mathrm{~K} 2 \mathrm{C} 1, \mathrm{~K} 1 \mathrm{C} 9, \mathrm{~K} 22 \mathrm{E}$ and K1C16.

Proteomic alterations of AH associated with cataract development. According to the differently expressed proteins between the above-mentioned three groups of patients and their respective controls, the present study further determined the proteomic alterations of $\mathrm{AH}$ associated with cataract development. As demonstrated in Table II, 44 proteins were identified to be associated with cataract development, which were markedly altered in all three groups of patients. Among these proteins, eight proteins were synchronously downregulated and 33 proteins were synchronously upregulated in the patients with high myopia, glaucoma or diabetes. Of the eight downregulated proteins,
$\operatorname{Ig} \mu$ chain $\mathrm{C}$ region was decreased the most, which is known to be important in primary defense mechanisms (28). The other two most decreased proteins were K22E and $\mathrm{K} 1 \mathrm{C} 10$, with average fold-changes of $\sim 0.18$ and 0.30 , respectively. By contrast, $\beta$-crystallin A4 and $\alpha$-crystallin A were upregulated. The remaining three proteins were downregulated or upregulated in only one or two groups of patients. For example, the levels of K1C16 were higher in patients with high myopia and those with glaucoma, but were lower in patients with diabetes.

Validation of alterations in protein expression using ELISA analysis. To confirm the alterations in protein expression demonstrated by the iTRAQ analysis, ELISA analysis was used to examine the levels of five AH proteins (CRDL1, APLP2, F6SYF8, CP2S1 and F8W7M9), which were randomly selected from Table II. The results of the ELISA showed that the alterations in the five differently expressed proteins were all consistent with the proteomics data obtained using iTRAQ analysis (Fig. 3A-E). 


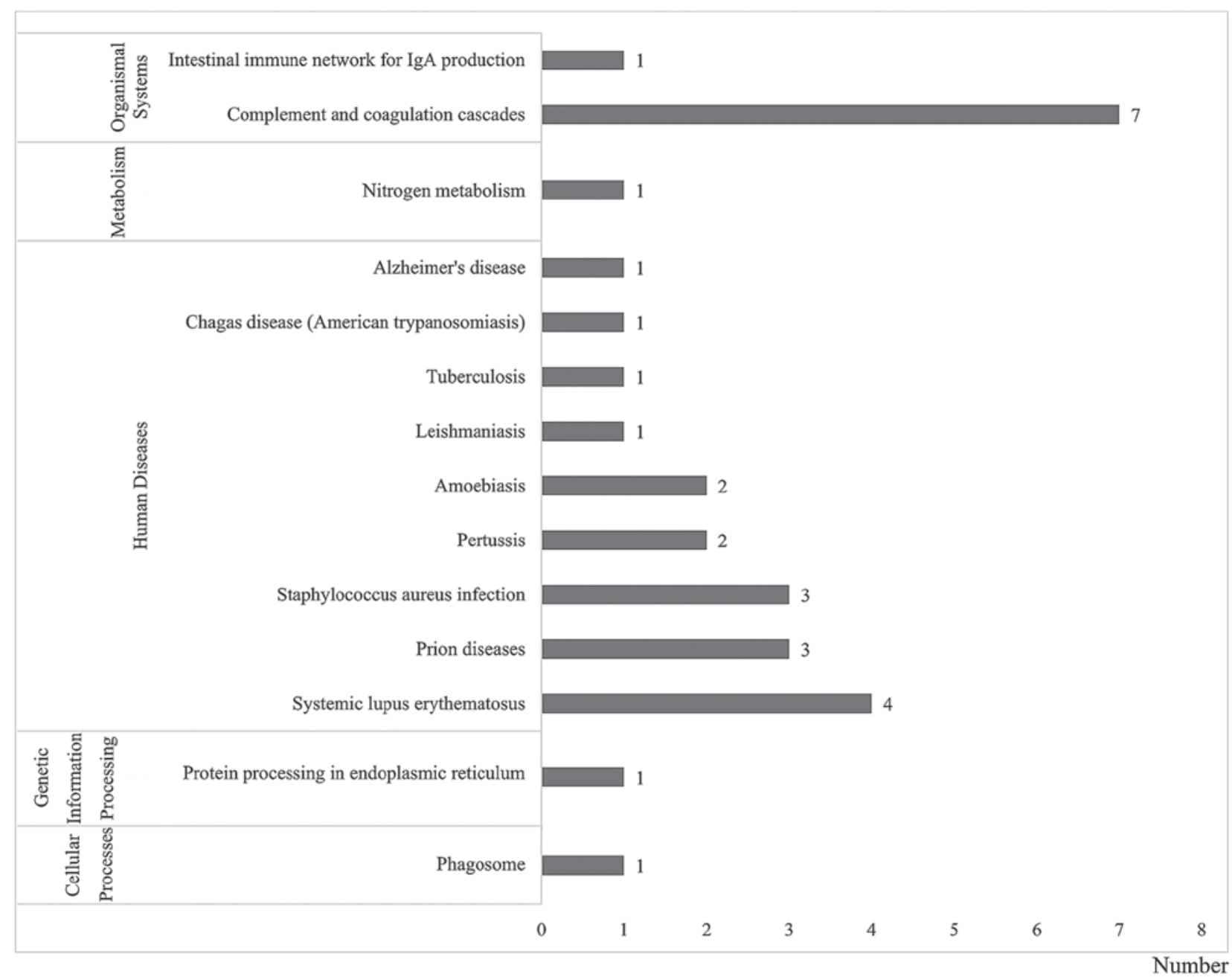

Figure 4. Pathway annotation analysis of proteins associated with cataract development.

Functional analysis of cataract-associated proteins. To obtain further insight into the functions of cataract-associated proteins, GO annotation analysis of the 44 differentially expressed proteins was performed, which were grouped into three functional groups: Cellular component, molecular function and biological process. For the cellular component ontology, there were nine GO terms, including cell part, extracellular region part, macromolecular complex, organelle, organelle part, extracellular region, membrane-enclosed lumen, synapse and synapse part. For the molecular function ontology, seven GO terms were identified, including binding, enzyme regulator activity, structural molecule activity and catalytic activity. For biological processes, the 44 GO terms were determined and included the regulation of biological process, metabolic process, response to stress, developmental process and establishment of localization functions. In particular, 17 proteins were involved in terms of binding for function ontology, whereas for biological processes, 19, 13 and 13 proteins were involved in the terms regulation of biological process, metabolic process and response to stress, respectively.

Pathway annotation analysis was also performed to determine significant metabolic and/or signal transduction pathways (Fig. 4). The results showed that there were 14 significantly biological pathways, which belonged to five groups: Cellular processes, genetic information processing, human diseases, metabolism and organismal systems. Of these 14 pathways, 23 proteins were involved in infectious diseases, neurodegenerative diseases, signal transduction, immune system, and transport and catabolism.

Bioinformatics analysis using the STRING (version 9.0) database was used to search known evidence of physical/functional interactions between the identified cataract-associated proteins. As demonstrated in Fig. 5, three groups comprising 11 proteins were determined in the biological networks. Among each group, the confidence between any two proteins was $>0.4$. The first notable group contained seven proteins, including afamin (AFM), $\alpha$-2-HS-glycoprotein (AHSG), $\alpha$-1-antitrypsin (SERPINA1), $\alpha$-1-antichymotrypsin (SERPINA3), apolipoprotein E (APOE), apolipoprotein A-II (APOA2) and apolipoprotein C-III (APOC3). A number of their interactions were high-level, including the interaction between APOE and APOC3C3 and C5 (score 0.997), and the interaction between APOE and APOA2 (score 0.991). In addition, two proteins (C3 and C5) were grouped, which belong to proteins of the immune system with a central role in the complement system and contribution to innate immunity (29). In addition, the other two proteins (fibrinogen $\beta$ and fibrinogen $\gamma$ ) interacted with 


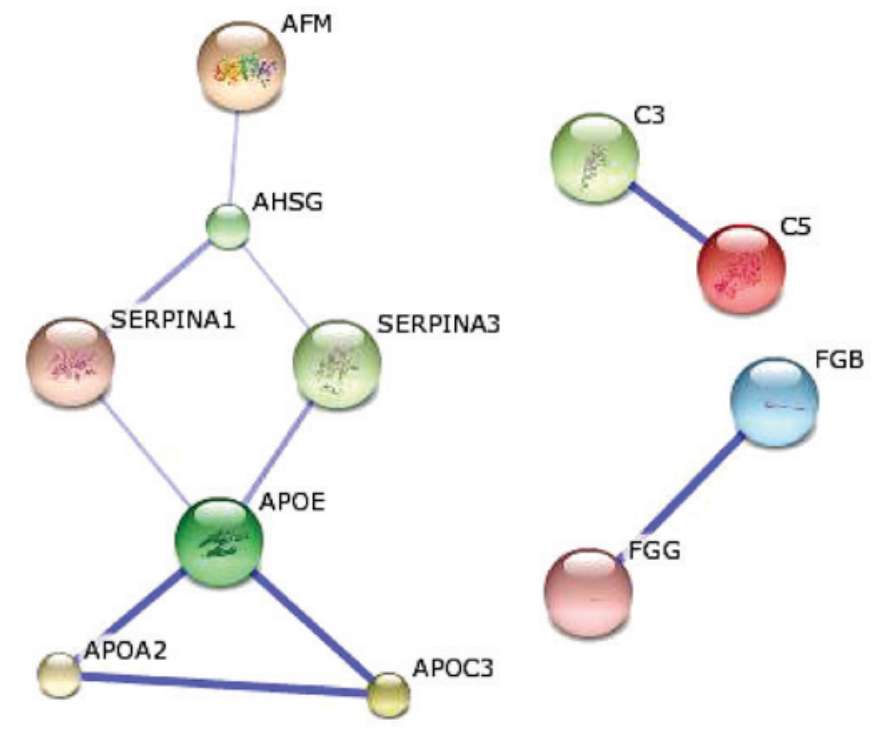

Figure 5. Biological networks regulated by the identified cataract-associated proteins. The blue line represents the positive confidence between two proteins. The deeper the blue color, the higher the confidence. AFM, afamin; AHSG, $\alpha$-2-HS-glycoprotein; SERPINA1, $\alpha$-1-antitrypsin, SERPINA3, $\alpha$-1-antichymotrypsin; APOE, apolipoprotein E; APOA2, apolipoprotein A-II; APOC3, apolipoprotein C-III; FGB, fibrinogen $\beta$; FGG, fibrinogen $\gamma$.

each other (scoring 0.797), which were involved in blood clot formation and may contribute to the pathology of thrombosis.

\section{Discussion}

As cataracts are the primary cause of blindness and visual impairment, an increasing number of studies have focused on proteomic analyses of ocular fluids to elucidate the mechanism. Among these ocular fluids, the $\mathrm{AH}$ is important for the eye in maintaining certain functions, including refraction, shape and intraocular pressure (17). Although proteomic analyses of cataract development have been performed in AH, questions regarding the mechanism of cataract formation remain due to the lack of integrative studies (30-33). In addition, previous studies on high myopia or glaucoma-associated cataracts were performed using traditional 2-DE analysis, which usually results in lower levels of accuracy due to the lower concentration of proteins in the $\mathrm{AH}$. By subjecting samples to a high throughput protein identification technique, labeled by iTRAQ, the present study compared the AH proteome of three groups of patients and their respective controls, which revealed significant variation, not only in proteins levels, but in protein functions. The proteomic differences in the AH among patients and controls were then determined to be associated with cataract development. Additional protein-protein interactions were also revealed, and the differences in five randomly selected proteins were confirmed using ELISA assays. Thus, the present study not only broadened the proteomic profile of $\mathrm{AH}$, compared with previous studies, but provided novel insights into protein alterations and their functions in cataract development (30-33). The results of the present study assist in further extending the current understanding of cataract formation resulting from high myopia, glaucoma or diabetes, and may be useful for providing potential prevention and treatment strategies.
Myopia is the most common visual impairment in the world, and the worldwide prevalence of high myopia has been estimated to be between 0.3 and $9.6 \%$. The percentage has recently been reported to be as high as $16 \%$ in some Asian countries (30). High myopia is usually defined as myopia $>-6.00$ diopters or with an axis length $\geq 26 \mathrm{~mm}$. It is also termed pathological myopia as it may present with retinal pathological alterations in the posterior pole with progressive choroidal degeneration, in addition to other complications. Increasing clinical evidence has revealed that high myopia is a risk factor for cataracts and their association is well established. For example, a previous study by Lim et al (1999) suggested that early-onset myopia may be a reliable and independent risk factor for PSC cataracts, and high myopia has been associated with nuclear and PSCs (34). In another previous study, the results showed that high myopia is a risk factor for dark nuclear cataracts (35). The results revealed an increased oxygen tension by earlier vitreous liquefaction and the downregulation of $\alpha$ A-crystallin, a major component of antioxidative mechanisms within the lens, which functions in the pathogenesis of high-myopic dark nuclear cataracts. In addition, Duan et al (36) used 2-DE to identify disease-specific proteins in the $\mathrm{AH}$ between proteomes in patients with cataracts (control) and those with high myopia. The results indicated higher total protein concentrations, and the levels of six proteins, including albumin, transthyretin and a vitamin D-binding protein, were significantly increased in AH with high myopia, compared with the non-myopia group. In the present study, 146 proteins were differently expressed between patients with high myopia and controls. These results may provide potential biomarkers for cataract development due to high myopia and extend current understanding on the underlying mechanisms at the proteomic level.

Excluding high myopia, previous studies have revealed that cataract progression is frequently associated with glaucoma, and there have been studies focusing on proteomic differences between patients with various types of glaucoma and controls (5,36-38). These studies revealed several potential biomarkers, which may be useful in the development of novel diagnostic markers and therapeutic targets. For example, González-Iglesias et al (2014) used several techniques in a comprehensive proteomic workflow to determine the alterations in sera proteins between patients with POAG, pseudoexfoliation glaucoma (PEXG) and healthy controls (5). Their study offered novel perspectives in the identification of glaucoma biomarkers in the serum of patients with POAG and PEXG, which were considered to be useful for the clinical prediction, prognosis, diagnosis and monitoring of POAG and PEXG on a large scale. However, there has been minimal reporting on proteomic alterations with glaucoma. In the present study, the proteomic differences between patients with glaucoma and their controls were compared, and 264 proteins were differently expressed, which may be involved in cataract development. These results may provide potential biomarkers for the monitoring of cataract formation associated with glaucoma.

Diabetes has been reported to be associated with a 5-fold higher prevalence of cataracts and is a major cause of blindness worldwide (39). Proteomic studies on characterizing lenticular proteins and elucidating the complex factors 
involved in the development of diabetic cataracts have been performed $(14,40)$. For example, by using 2-D differential in-gel electrophoresis coupled with MS, Su et al (2014) identified differential alterations in proteins and metabolites underlying 'fast' type 1 and 'slow' type 2 diabetic cataract formation in rats (14). The results revealed alterations in the abundance of crystallins and alterations in HMW noncrystallin proteins, which assisted to in elucidating the shared and differential pathological mechanisms associated with the two types of diabetic cataracts. Similarly, Zhu et al (2013) used 2-DE, MS, and ELISA to investigate the differential lens proteomics between diabetic cataracts, age-related cataracts and normal subjects (40). The results reflected the differential proteins in diabetic and age-related cataract lenses, compared with normal subjects. In the present study, the proteomic differences between patients with diabetes and their controls were also compared using a high throughput technology, rather than traditional 2-DE technology, which revealed 130 proteins were differently expressed. These results may provide potential biomarkers for the monitoring of cataract formation associated with diabetes, and may be assist with further investigations on the principles and mechanisms of cataract development.

To the best of our knowledge, cataracts usually result from dysfunction of the lens, where the proteins are photo-oxidatively damaged, aggregated and accumulated in lens opacities (41). The results of the present study demonstrated that there were proteomic alterations among the three above-mentioned groups of patients, and 44 proteins were altered, which were considered to be cataract-associated proteins. Further functional analyses of the 44 identified cataract-associated proteins were performed, and 14 pathways were annotated, including infectious diseases, neurodegenerative diseases, signal transduction, signaling molecules and interaction, and transport and catabolism. In addition, physical/functional interactions between the identified cataract-associated proteins indicated that 11 proteins in the three groups were involved in the established biological network. In particular, four crystallins and heat shock protein $\beta-1$, were reported to be connected with nervous system disease and eye disease, including cataracts.

In conclusion, the proteomic profiling data obtained in the present study revealed marked proteomic differences between the three groups of patients (high myopia, glaucoma and diabetes) and their corresponding controls. Based on these differences, 44 cataract-associated proteins were determined, and five of these 44 cataract-associated proteins were randomly selected and confirmed using ELISA assays. The biological functions and interaction networks of these 44 proteins were also identified. The results provide novel insights into the mechanism of cataract development, and potential biomarkers for the diagnosis and monitoring of cataract formation.

\section{Acknowledgements}

The present study was supported by the Research Project of Health and Family Planning Commission in Shanghai (grant no. 20124108).

\section{References}

1. Pascolini D and Mariotti SP: Global estimates of visual impairment: 2010. Br J Ophthalmol 96: 614-618, 2012.

2. Korsakova NV, Sergeeva VE and Petrov SB: Immunohisto chemical analysis of lens cells on formation of different types of age-related cataract in humans. Neurosci Behav Physiol 38: 887-890, 2008

3. Salm M, Belsky D and Sloan FA: Trends in cost of major eye diseases to medicare, 1991 to 2000. Am J Ophthalmol 142: 976-982, 2006.

4. Sachdeva R, Sears JE and Rychwalski PJ: A novel case of bilateral high myopia, cataract and total retinal detachment associated with interstitial 11q deletion. Ophthalmic Genet 31: 84-88, 2010.

5. González-Iglesias H, Álvarez L, García M, Escribano J, Rodríguez-Calvo PP, Fernández-Vega L and Coca-Prados M: Comparative proteomic study in serum of patients with primary open-angle glaucoma and pseudoexfoliation glaucoma. J Proteomics 98: 65-78, 2014.

6. Izzotti A, Longobardi M, Cartiglia C and Saccà SC: Proteome alterations in primary open angle glaucoma aqueous humor. J Proteome Res 9: 4831-4838, 2010.

7. Ji Y, Cai L, Zheng T, Ye H, Rong X, Rao J and Lu Y: The mechanism of UVB irradiation induced-apoptosis in cataract. Mol Cell Biochem 401: 87-95, 2015.

8. Khan L, Khan RA, Ahmed W, Rauf A, Khan MW, Khan W, Durrani SA and Qayum S: Frequency, causes and cutting-edge treatment of cataract: A review. AJBLS 3: 25-28, 2015.

9. Foster PJ, Wong TY, Machin D, Johnson GJ and Seah SK: Risk factors for nuclear, cortical and posterior subcapsular cataracts in the Chinese population of Singapore: The tanjong pagar survey. Br J Ophthalmol 87: 1112-1120, 2003.

10. Cheng CY, Liu JH, Chen SJ and Lee FL: Population-based study on prevalence and risk factors of age-related cataracts in Peitou, Taiwan. Zhonghua Yi Xue Za Zhi (Taipei) 63: 641-648, 2000.

11. Pan CW, Boey PY, Cheng CY, Saw SM, Tay WT, Wang JJ, Tan AG, Mitchell P and Wong TY: Myopia, axial length and age-related cataract: The Singapore Malay eye study. Invest Ophthalmol Vis Sci 54: 4498-4502, 2013.

12. Chiang SY, Tsai ML, Wang CY, Chen A, Chou YC, Hsia CW, Wu YF, Chen HM, Huang TH, Chen PH, et al: Proteomic analysis and identification of aqueous humor proteins with a pathophysiological role in diabetic retinopathy. J Proteomics 75: 2950-2959, 2012

13. Hadjistilianou T, Giglioni S, Micheli L, Vannoni D, Brogi E, Cevenini G, Cortelazzo A, De Francesco S, Menicacci F and Leoncini R: Analysis of aqueous humour proteins in patients with retinoblastoma. Clin Exp Ophthalmol 40: e8-e15, 2012.

14. Su S, Leng F, Guan L, Zhang L, Ge J, Wang C, Chen S and Liu P: Differential proteomic analyses of cataracts from rat models of type 1 and 2 diabetes. Invest Ophthalmol Vis Sci 55: 7848-7861, 2014.

15. Zhou HY, Yan H, Wang LL, Yan WJ, Shui YB and Beebe DC: Quantitative proteomics analysis by iTRAQ in human nuclear cataracts of different ages and normal lens nuclei. Proteomics Clin Appl 9: 776-786, 2015.

16. Monteiro JP, Santos FM, Rocha AS, Castro-de-Sousa JP, Queiroz JA, Passarinha LA and Tomaz CT: Vitreous humor in the pathologic scope: Insights from proteomic approaches. Proteomics Clin Appl 9: 187-202, 2015.

17. Grus FH, Joachim SC and Pfeiffer N: Proteomics in ocular fluids. Proteomics Clin Appl 1: 876-888, 2007.

18. Wu WW, Wang G, Baek SJ and Shen RF: Comparative study of three proteomic quantitative methods, DIGE, cICAT and iTRAQ, using 2D gel-or LC-MALDI TOF/TOF. J Proteome Res 5: 651-658, 2006.

19. Karbassi M, Khu PM, Singer DM and Chylack LT Jr: Evaluation of lens opacities classification system III applied at the slitlamp. Optom Vis Sci 70: 923-928, 1993

20. Bennett KL, Funk M, Tschernutter M, Breitwieser FP, Planyavsky M, Ubaida Mohien C, Müller A, Trajanoski Z, Colinge J, Superti-Furga G and Schmidt-Erfurth U: Proteomic analysis of human cataract aqueous humour: Comparison of one-dimensional gel LCMS with two-dimensional LCMS of

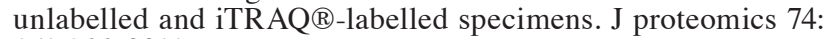
151-166, 2011.

21. Qianqian Y, Yong Y, Zhaodong C, Yonghui T, Jun S and Yuzheng H: Differential protein expression between type 1 diabetic cataract and age-related cataract patients. Folia Biol (Praha) 61: 74-80, 2015. 
22. Omenn GS: The human eye proteome project. Proteomics 13: 2375-2376, 2013.

23. Jiang CK, Magnaldo T, Ohtsuki M, Freedberg IM, Bernerd F and Blumenberg M: Epidermal growth factor and transforming growth factor alpha specifically induce the activation- and hyperproliferation-associated keratins 6 and 16. Proc Natl Acad Sci USA 90: 6786-6790, 1993.

24. Héon E, Priston M, Schorderet DF, Billingsley GD, Girard PO, Lubsen N and Munier FL: The gamma-crystallins and human cataracts: A puzzle made clearer. Am J Hum Genet 65: 1261-1267, 1999.

25. Hejtmancik JF, Wingfield PT and Sergeev YV: Beta-crystallin association. Exp Eye Res 79: 377-383, 2004.

26. Brown RH, Zhong L and Lynch MG: Lens-based glaucoma surgery: Using cataract surgery to reduce intraocular pressure. J Cataract Refract Surg 40: 1255-1262, 2014.

27. Lee AY and Chung SS: Contributions of polyol pathway to oxidative stress in diabetic cataract. FASEB J 13: 23-30, 1999.

28. Tisch R, Roifman CM and Hozumi N: Functional differences between immunoglobulins $M$ and $D$ expressed on the surface of an immature B-cell line. Proc Natl Acad Sci USA 85: 6914-6918, 1988.

29. De Bruijn MH and Fey GH: Human complement component C3: CDNA coding sequence and derived primary structure. Proc Natl Acad Sci USA 82: 708-712, 1985.

30. Duan X, Lu Q, Xue P, Zhang H, Dong Z, Yang F and Wang N: Proteomic analysis of aqueous humor from patients with myopia. Mol Vis 14: 370-377, 2008.

31. Chowdhury UR, Madden BJ, Charlesworth MC and Fautsch MP: Proteome analysis of human aqueous humor. Invest Ophthalmol Vis Sci 51: 4921-4931, 2010.

32. Richardson MR, Segu ZM, Price MO, Lai X, Witzmann FA, Mechref Y, Yoder MC and Price FW: Alterations in the aqueous humor proteome in patients with fuchs endothelial corneal dystrophy. Mol Vis 16: 2376-2383, 2010.
33. Yao J, Liu X, Yang Q, Zhuang M, Wang F, Chen X, Hang H, Zhang $\mathrm{W}$ and Liu Q: Proteomic analysis of the aqueous humor in patients with wet age-related macular degeneration. Proteomics Clin Appl 7: 550-560, 2013.

34. Lim R, Mitchell P and Cumming RG: Refractive associations with cataract: The blue mountains eye study. Invest Ophthalmol Vis Sci 40: 3021-3026, 1999.

35. Zhu XJ, Zhou P, Zhang KK, Yang J, Luo Y and Lu Y: Epigenetic regulation of $\alpha \mathrm{A}$-crystallin in high myopia-induced dark nuclear cataract. PLoS One 8: e81900, 2013.

36. Duan X, Xue P, Wang N, Dong Z, Lu Q and Yang F: Proteomic analysis of aqueous humor from patients with primary open angle glaucoma. Mol Vis 16: 2839-2846, 2010.

37. BouhenniRA, Al Shahwan S, Morales J, Wakim BT, Chomyk AM, Alkuraya FS and Edward DP: Identification of differentially expressed proteins in the aqueous humor of primary congenital glaucoma. Exp Eye Res 92: 67-75, 2011.

38. Saccà SC, Centofanti M and Izzotti A: New proteins as vascular biomarkers in primary open angle glaucomatous aqueous humor. Invest Ophthalmol Vis Sci 53: 4242-4253, 2012.

39. Obrosova IG, Chung SS and Kador PF: Diabetic cataracts: Mechanisms and management. Diabetes Metab Res Rev 26: 172-180, 2010.

40. Zhu J, Shao J, Yao Y, Chu ZD, Yu QQ, Zhao W, Lin Q and Zhang ZY: Differential proteomics analysis of proteins from human diabetic and age-related cataractous lenses. J Res Med Sci 18: 984-989, 2013.

41. Kyselova Z: Mass spectrometry-based proteomics approaches applied in cataract research. Mass Spectrom Rev 30: 1173-1184, 2011. 